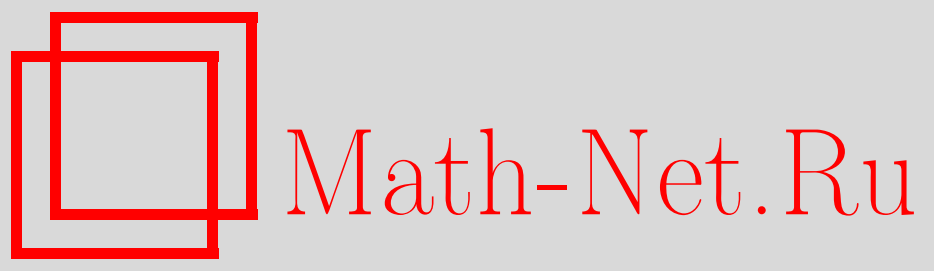

T. Х. Расулов, Асимптотика дискретного спектра одного модельного оператора, ассоциированного с системой трех частиц на решетке, ТМФ, 2010, том 163, номер 1, 34-44

DOI: https://doi.org/10.4213/tmf6485

Использование Общероссийского математического портала Math-Net.Ru подразумевает, что вы прочитали и согласны с пользовательским соглашением http: //www . mathnet.ru/rus/agreement

Параметры загрузки:

IP : 3.82 .47 .9

26 апреля 2023 г., 03:32:27

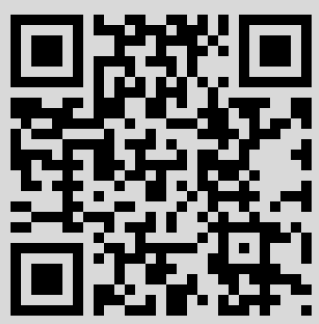




\section{АСИМПТОТИКА ДИСКРЕТНОГО СПЕКТРА ОДНОГО МОДЕЛЬНОГО ОПЕРАТОРА, АССОЦИИРОВАННОГО С СИСТЕМОЙ ТРЕХ ЧАСТИЦ НА РЕШЕТКЕ}

\footnotetext{
Рассматривается модельный оператор Шредингера $H_{\mu}$, ассоциированный с системой трех частиц на трехмерной решетке $\mathbb{Z}^{3}$, с параметром-функцией специального вида. Доказано, что если соответствующая модель Фридрихса имеет резонанс с нулевой энергией, то у оператора $H_{\mu}$ существует бесконечное число отрицательных собственных значений, накапливающихся в нуле (эффект Ефимова). Получена асимптотика числа собственных значений оператора $H_{\mu}$, лежащих ниже $z$, при $z \rightarrow-0$.
}

Ключевые слова: модельный оператор, модель Фридрихса, принцип Бирмана-Швингера, эффект Ефимова, оператор Гильберта-Шмидта, резонанс с нулевой энергией, дискретный спектр.

\section{1. ВВЕДЕНИЕ}

В спектральной теории непрерывного и решетчатого трехчастичных операторов Шредингера имеется следующий замечательный результат: если в системе трех частиц, взаимодействие которых описывается парными короткодействующими потенциалами, ни одна из трех двухчастичных подсистем не имеет связанных состояний с отрицательной энергией, но по меньшей мере две из этих систем имеют резонанс с нулевой энергией, то у этой трехчастичной системы существует бесконечное число связанных состояний с отрицательными энергиями, накапливающимися в нуле. Этот эффект впервые был обнаружен Ефимовым [1] и в случае непрерывного оператора Шредингера подробно обсуждался во многих работах (см., например, статьи [2]-[4]). Основным результатом работы [3] является асимптотика вида $\mathcal{U}_{0}|\ln | \lambda||$ для числа собственных значений трехчастичного непрерывного оператора Шредингера, лежащих левее $\lambda<0$, где коэффициент $\mathcal{U}_{0}>0$ зависит только от частных масс трех частиц (см. также работу [4]). Недавно в работе [5] было доказано наличие эффекта Ефимова в $N$-частичной квантовой системе, $N \geqslant 4$, и получена оценка снизу числа собственных значений полного гамильтониана. Для системы трех частиц на решетке существование эффекта Ефимова обнаружено в работах [6], [7],

*Бухарский государственный университет, Бухара, Узбекистан. E-mail: rth@mail.ru 
а строгое доказательство изложено в работах [8], [9], а затем в работах [10]-[12], причем в статьях [11], [12] получена аналогичная непрерывному случаю асимптотика дискретного спектра системы трех частиц на решетке.

В настоящей работе рассматривается модельный оператор $H_{\mu}=H_{0}-\mu V_{1}-\mu V_{2}$, $\mu>0$, ассоциированный с системой трех частиц на трехмерной решетке $\mathbb{Z}^{3}$, в случае специального вида функции $w$, являющейся параметром оператора $H_{0}$ (см. ниже формулы $(1),(2))$. Эта функция имеет невырожденный минимум в $n>1$ различных точках шестимерного тора. Доказано, что если модель Фридрихса имеет резонанс с нулевой энергией, то число отрицательных собственных значений оператора $H_{\mu}$ бесконечно, и эти собственные значения накапливаются в нуле; кроме того, получена асимптотика для числа $N_{\mu}(z)$ собственных значений оператора $H_{\mu}$, лежащих ниже $z<0$. Следует отметить, что спектр и асимптотика дискретного спектра несимметричного модельного оператора $H_{\mu}$ изучен в [13] в случае произвольной функции $w$ с $n=1$ невырожденным минимумом. Асимптотика, полученная в настоящей работе, является обобщением асимптотик из работ [3], [4], [11]-[13].

Структура статьи такова. В разделе 2 вводится модельный оператор Шредингера $H_{\mu}$, который рассматривается как ограниченный самосопряженный оператор в гильбертовом пространстве, и формулируется основной результат работы. В разделе 3 изучаются некоторые спектральные свойства модели Фридрихса, которые играют важную роль при анализе дискретного спектра оператора $H_{\mu}$. В разделе 4 для оператора $H_{\mu}$ получено утверждение, отражающее известный принцип Бирмана-Швингера. Раздел 5 посвящен доказательству основного результата.

\section{2. МОДЕЛЬНЫЙ ОПЕРАТОР И ФОРМУЛИРОВКА ОСНОВНОГО РЕЗУЛЬТАТА}

Пусть $\mathbf{T}^{3}=(-\pi ; \pi]^{3}$ - трехмерный куб с соответствующим отождествлением противоположных граней. Мы рассматриваем $\mathbf{T}^{3}$ как абелеву группу, в которой операции сложения и умножения на вещественное число введены как операции сложения и умножения на вещественное число в $\mathbb{R}^{3}$ по модулю $(2 \pi \mathbb{Z})^{3}$. Пусть $\left(\mathbf{T}^{3}\right)^{2}=\mathbf{T}^{3} \times \mathbf{T}^{3}$ - декартово произведение, $L_{2}\left(\mathbf{T}^{3}\right)$ - гильбертово пространство квадратично-интегрируемых (комплекснозначных) функций, определенных на $\mathbf{T}^{3}$, и $L_{2}^{\mathrm{s}}\left(\left(\mathbf{T}^{3}\right)^{2}\right)$ - гильбертово пространство квадратично-интегрируемых симметричных (комплекснозначных) функций, определенных на $\left(\mathbf{T}^{3}\right)^{2}$.

Рассмотрим следующий модельный оператор $H_{\mu}$ в пространстве $L_{2}^{\mathrm{s}}\left(\left(\mathbf{T}^{3}\right)^{2}\right)$ :

$$
H_{\mu}=H_{0}-\mu V_{1}-\mu V_{2},
$$

где действие операторов $H_{0}$ и $V_{i}, i=1,2$, определяется по формулам

$$
\begin{aligned}
& \left(H_{0} f\right)(p, q)=w(p, q) f(p, q), \\
& \left(V_{1} f\right)(p, q)=\varphi(p) \int_{\mathbf{T}^{3}} \varphi(s) f(s, q) d s \\
& \left(V_{2} f\right)(p, q)=\varphi(q) \int_{\mathbf{T}^{3}} \varphi(s) f(p, s) d s .
\end{aligned}
$$


Здесь $\mu$ - положительное действительное число, $\varphi(\cdot)$ - вещественно-аналитическая, четная функция на $\mathbf{T}^{3}$, а функция $w(\cdot, \cdot)$ имеет вид

$$
\begin{gathered}
w(p, q)=\varepsilon(p)+\varepsilon(p+q)+\varepsilon(q), \\
\varepsilon(p)=\sum_{j=1}^{3}\left(1-\cos \left(m p^{(j)}\right)\right), \quad p=\left(p^{(1)}, p^{(2)}, p^{(3)}\right) \in \mathbf{T}^{3}, \quad m \in \mathbb{N} .
\end{gathered}
$$

При этих предположениях оператор $H_{\mu}$ является ограниченным и самосопряженным в $L_{2}^{\mathrm{s}}\left(\left(\mathbf{T}^{3}\right)^{2}\right)$.

Для формулировки основного результата введем оператор $h_{\mu}(p)$ модели Фридрихса, действующий в $L_{2}\left(\mathbf{T}^{3}\right)$, как $h_{\mu}(p)=h_{0}(p)-\mu v$, где операторы $h_{0}(p)$ и $v$ определяются по правилам

$$
\left(h_{0}(p) f_{1}\right)(q)=w(p, q) f(q), \quad(v f)(q)=\varphi(q) \int_{\mathbf{T}^{3}} \varphi(s) f(s) d s, \quad p \in \mathbf{T}^{3} .
$$

Возмущение $\mu v$ оператора $h_{0}(p)$ является самосопряженным одномерным оператором. Из известной теоремы Вейля [14] о сохранении существенного спектра при возмущениях конечного ранга вытекает, что существенный спектр $\sigma_{\text {ess }}\left(h_{\mu}(p)\right)$ оператора $h_{\mu}(p)$ совпадает с существенным спектром оператора $h_{0}(p)$. Известно, что $\sigma_{\mathrm{ess}}\left(h_{0}(p)\right)=[m(p) ; M(p)]$, где числа $m(p)$ и $M(p)$ определяются равенствами

$$
m(p)=\varepsilon(p)+2 \sum_{j=1}^{3}\left(1-\cos \frac{m p^{(j)}}{2}\right), \quad M(p)=\varepsilon(p)+2 \sum_{j=1}^{3}\left(1+\cos \frac{m p^{(j)}}{2}\right) .
$$

Следовательно, $\sigma_{\mathrm{ess}}\left(h_{\mu}(p)\right)=[m(p) ; M(p)]$.

Следующая теорема описывает положение существенного спектра оператора $H_{\mu}$.

Теорема 1 [13]. Существенный спектр оператора $H_{\mu}$ имеет вид

$$
\sigma_{\mathrm{ess}}\left(H_{\mu}\right)=\bigcup_{p \in \mathbf{T}^{3}}\left\{\sigma_{\mathrm{disc}}\left(h_{\mu}(p)\right) \bigcup\left[0 ; \frac{27}{2}\right]\right\}
$$

где через $\sigma_{\mathrm{disc}}\left(h_{\mu}(p)\right)$ обозначен дискретный спектр оператора $h_{\mu}(p)$.

ОПРЕДЕЛЕНИЕ 1. Множества $\bigcup_{p \in \mathbf{T}^{3}} \sigma_{\text {disc }}\left(h_{\mu}(p)\right)$ и $[0 ; 27 / 2]$ называются соответственно двухчастичной и трехчастичной ветвями существенного спектра оператора $H_{\mu}$.

Обозначим через $n \equiv n(m)$ число точек $\left(p_{i}, q_{j}\right) \in\left(\mathbf{T}^{3}\right)^{2}, p_{i}=\left(p_{i}^{(1)}, p_{i}^{(2)}, p_{i}^{(3)}\right)$, $q_{j}=\left(q_{j}^{(1)}, q_{j}^{(2)}, q_{j}^{(3)}\right)$, для которых

$$
\begin{gathered}
p_{i}^{(k)}, q_{j}^{(k)} \in\left\{0, \pm \frac{2}{m} \pi, \pm \frac{4}{m} \pi, \ldots, \pm \frac{m^{\prime}}{m} \pi\right\}, \quad k=1,2,3 \\
p_{s} \neq p_{l}, \quad q_{s} \neq q_{l} \quad \text { при } \quad s \neq l ;
\end{gathered}
$$

здесь

$$
m^{\prime}= \begin{cases}m-2, & \text { если } m-\text { четное число, } \\ m-1, & \text { если } m-\text { нечетное число. }\end{cases}
$$


Можно легко проверить, что $n=\left(m^{\prime}+1\right)^{6}$ и функция $w(\cdot, \cdot)$ имеет невырожденный минимум в точках $\left(p_{i}, q_{j}\right) \in\left(\mathbf{T}^{3}\right)^{2}$. Дополнительно будем предполагать, что $m \geqslant 3$ (если $m=1,2$, то $n=1$, а в настоящей работе нам интересен случай $n>1$ ).

ЗАМЕчАНИЕ 1. При изучении дискретного спектра оператора $H_{\mu}$ основную роль играют нули функции $\varphi(\cdot)$ в точках $q_{j} \in \mathbf{T}^{3}, j=\overline{1, \sqrt{n}}$ (см., например, [13]). Предположим, что только в $n_{0}$ точках $\left(1<n_{0} \leqslant \sqrt{n}\right)$ множества $\left\{q_{j}\right\}_{j=1}^{\sqrt{n}}$ функция $\varphi(\cdot)$ отлична от нуля. Пусть

$$
\mathbf{n}=n_{0} \sqrt{n} .
$$

Рассмотрим множество $\left\{\left(p_{s_{i}}, q_{s_{i}}\right) \in\left(\mathbf{T}^{3}\right)^{2}: i=\overline{1, n}\right\}$, где $s_{i}=\overline{1, n}$, такое, что $\varphi\left(q_{s_{i}}\right) \neq 0$ при $i=\overline{1, \mathbf{n}}$ и $\varphi\left(q_{s_{i}}\right)=0$ при $i=\overline{\mathbf{n}+1, n}$. Здесь $\left(p_{s_{i}}, q_{s_{i}}\right), i=\overline{1, n},-$ точки множества, заданного в (3), перенумерованные в соответствии с правилом

$$
p_{s_{k-1} \sqrt{n}+j}=p_{k}, \quad k, j=\overline{1, n} ; \quad q_{s_{k-1} \sqrt{n_{0}}+j}=q_{j}, \quad k, j=\overline{1, n_{0}} .
$$

Другими словами, если расположить точки $\left(p_{i}, q_{j}\right)_{i, j=1}^{\sqrt{n}}$ в виде квадратной таблицы

$$
\begin{array}{cccccc}
\left(p_{1}, q_{1}\right) & \left(p_{1}, q_{2}\right) & \ldots & \left(p_{1}, q_{n_{0}}\right) & \ldots & \left(p_{1}, q_{\sqrt{n}}\right) \\
\left(p_{2}, q_{1}\right) & \left(p_{2}, q_{2}\right) & \ldots & \left(p_{2}, q_{n_{0}}\right) & \ldots & \left(p_{2}, q_{\sqrt{n}}\right) \\
\vdots & \vdots & \ddots & \vdots & \ddots & \vdots \\
\left(p_{\sqrt{n}}, q_{1}\right) & \left(p_{\sqrt{n}}, q_{2}\right) & \ldots & \left(p_{\sqrt{n}}, q_{n_{0}}\right) & \ldots & \left(p_{\sqrt{n}}, q_{\sqrt{n}}\right)
\end{array}
$$

то элементы левой части этой таблицы с номерами столбцов $j=\overline{1, n_{0}}, n_{0} \leqslant \sqrt{n}$, перечисляются слева направо вдоль строки и от верхней строки к нижней, а остальные в произвольном порядке.

ЗАмечание 2. Имеет место равенство $h_{\mu}\left(p_{s_{1}}\right) \equiv h_{\mu}\left(p_{s_{i}}\right), i=\overline{2, n}$.

Пусть $C\left(\mathbf{T}^{3}\right)\left(L_{1}\left(\mathbf{T}^{3}\right)\right)$ - банахово пространство непрерывных (соответственно, интегрируемых) функций на $\mathbf{T}^{3}$.

ОПРЕДЕЛЕНИЕ 2. Говорят, что оператор $h_{\mu}\left(p_{s_{1}}\right)$ имеет резонанс с нулевой энергией, если число 1 является собственным значением интегрального оператора $G_{\mu}$,

$$
\left(G_{\mu} \psi\right)(q)=\frac{\mu \varphi(q)}{2} \int_{\mathbf{T}^{3}} \frac{\varphi(s) \psi(s) d s}{\varepsilon(s)}, \quad \psi \in C\left(\mathbf{T}^{3}\right),
$$

и по крайней мере одна (с точностью до постоянного множителя) соответствующая собственная функция $\psi$ удовлетворяет условию $\psi\left(q_{s_{i}}\right) \neq 0, i=\overline{1, \mathbf{n}}$.

Если оператор $h_{\mu}\left(p_{s_{1}}\right)$ имеет резонанс с нулевой энергией, то функция

$$
f(q)=\frac{\mu \varphi(q)}{2 \varepsilon(q)} \in L_{1}\left(\mathbf{T}^{3}\right) \backslash L_{2}\left(\mathbf{T}^{3}\right)
$$

удовлетворяет уравнению $h_{\mu}\left(p_{s_{1}}\right) f=0$ (см. лемму 7 настоящей работы). 
Положим

$$
\mu_{0}=2\left(\int_{\mathbf{T}^{3}} \frac{\varphi^{2}(s) d s}{\varepsilon(s)}\right)^{-1} .
$$

Оператор $h_{\mu}\left(p_{s_{1}}\right)$ имеет резонанс с нулевой энергией тогда и только тогда, когда $\mu=\mu_{0}$ (см. лемму 2).

Обозначим через $\tau_{\text {ess }}\left(H_{\mu}\right)$ нижнюю грань существенного спектра $\sigma_{\text {ess }}\left(H_{\mu}\right)$ оператора $H_{\mu}$ и через $N_{\mu}(z)$ - число собственных значений (с учетом кратности) оператора $H_{\mu}$, лежащих левее точки $z<\tau_{\text {ess }}\left(H_{\mu}\right)$. Величина $\tau_{\text {ess }}\left(H_{\mu_{0}}\right)=0$, т.е. оператор $h_{\mu_{0}}(p), p \in \mathbf{T}^{3}$, не имеет отрицательных собственных значений (см. лемму 6).

Теперь сформулируем основной результат.

Теорема 2. Оператор $H_{\mu_{0}}$ имеет бесконечное число отрицательных собственных значений, накапливаюшихся в нуле, и для функиии $N_{\mu_{0}}(\cdot)$ справедливо равенство

$$
\lim _{z \rightarrow-0} \frac{N_{\mu_{0}}(z)}{|\ln | z||}=\frac{\mathbf{n} \gamma_{0}}{4 \pi},
$$

где число $\mathbf{n}$ определено в замечании 1 (см. формулу (4)) и число $\gamma_{0}$ есть единственный положительный корень уравнения

$$
\gamma \sqrt{3} \cos h \frac{\pi \gamma}{2}=8 \sin h \frac{\pi \gamma}{6}
$$

Видно, что в силу равенства (7) из положительности числа $\gamma_{0}$ вытекает, что число точек дискретного спектра оператора $H_{\mu_{0}}$ бесконечно. Отметим также, что асимптотика (7) является новым результатом и что аналогичная асимптотика не была получена для трехчастичного оператора Шредингера на $\mathbb{R}^{3}$ и $\mathbb{Z}^{3}$.

\section{3. СПЕКТРАЛЬНЫЕ СВОЙСТВА ОПЕРАТОРА $h_{\mu}(p)$}

В этом разделе мы рассмотрим некоторые спектральные свойства оператора модели Фридрихса $h_{\mu}(p), p \in \mathbf{T}^{3}$, которые играют важную роль при изучении дискретного спектра оператора $H_{\mu}$.

Пусть $\mathbb{C}$ - комплексная плоскость. Для любого $p \in \mathbf{T}^{3}$ определим аналитическую функцию $\Delta_{\mu}(p, \cdot)$ (детерминант Фредгольма, ассоциированный с оператором $\left.h_{\mu}(p)\right)$ :

$$
\Delta_{\mu}(p, z)=1-\mu \int_{\mathbf{T}^{3}} \frac{\varphi^{2}(q) d q}{w(p, q)-z}, \quad z \in \mathbb{C} \backslash \sigma_{\mathrm{ess}}\left(h_{\mu}(p)\right) .
$$

Теперь установим связь между собственными значениями оператора $h_{\mu}(p)$ и нулями функции $\Delta_{\mu}(p, \cdot)$.

Лемма 1 [13]. При каждом фиксированном $p \in \mathbf{T}^{3}$ оператор $h_{\mu}(p)$ имеет собственное значение $z \in \mathbb{C} \backslash \sigma_{\mathrm{ess}}\left(h_{\mu}(p)\right)$ тогда и только тогда, когда $\Delta_{\mu}(p, z)=0$.

Функция $w(\cdot, \cdot)$ имеет невырожденный минимум, равный нулю, в точках $\left(p_{s_{i}}, q_{s_{i}}\right) \in\left(\mathbf{T}^{3}\right)^{2}, i=\overline{1, n}$, и функция $\varphi(\cdot)$ является аналитической на $\mathbf{T}^{3}$, поэтому существует конечный интеграл

$$
\int_{\mathbf{T}^{3}} \frac{\varphi^{2}(q) d q}{w(p, q)}, \quad p \in \mathbf{T}^{3} .
$$


Из теоремы о предельном переходе под знаком интеграла Лебега и равенства $\Delta_{\mu}\left(p_{s_{i}}, 0\right)=\Delta_{\mu}\left(p_{s_{1}}, 0\right), i=\overline{2, n}$, следует, что

$$
\Delta_{\mu}\left(p_{s_{1}}, 0\right)=\lim _{p \rightarrow p_{s_{i}}} \Delta_{\mu}(p, 0), \quad i=\overline{1, n}
$$

Следующие три леммы играют важную роль при доказательстве основного результата, т.е. теоремы 2. Мы не приводим доказательство этих лемм, поскольку оно аналогично доказательству соответствующих лемм из работ [11], [13].

Лемма 2. Оператор $h_{\mu}\left(p_{s_{1}}\right)$ имеет резонанс с нулевой энергией тогда и только тогда, когда $\mu=\mu_{0}$.

Лемма 3. Имеет место разложение

$$
\Delta_{\mu_{0}}(p, z)=\frac{\pi^{2} \mu_{0}}{m^{2}} \sum_{j=1}^{\mathbf{n}} \varphi^{2}\left(q_{s_{j}}\right) \sqrt{\frac{3}{4}\left|p-p_{s_{i}}\right|^{2}-z}+O\left(\left|p-p_{s_{i}}\right|^{2}\right)+O(|z|)
$$

$n p u\left|p-p_{s_{i}}\right| \rightarrow 0, i=\overline{1, \mathbf{n}}, u z \rightarrow-0$.

Положим

$$
U_{\delta}\left(p_{0}\right)=\left\{p \in \mathbf{T}^{3}:\left|p-p_{0}\right|<\delta\right\}, \quad p_{0} \in \mathbf{T}^{3}, \quad \delta>0
$$

Лемма 4. Существуют числа $C_{1}, C_{2}, C_{3}>0 u \delta>0$ такие, что выполняются следующие неравенства:

$$
C_{1}\left|p-p_{s_{i}}\right|^{2} \leqslant\left|\Delta_{\mu_{0}}(p, 0)\right| \leqslant C_{2}\left|p-p_{s_{i}}\right|^{2}, \quad p \in U_{\delta}\left(p_{s_{i}}\right), \quad i=\overline{\mathbf{n}+1, n},
$$

$u\left|\Delta_{\mu_{0}}(p, 0)\right| \geqslant C_{3}$ для $p \in \mathbf{T}^{3} \backslash \bigcup_{i=1}^{n} U_{\delta}\left(p_{s_{i}}\right)$.

Из разложения

$$
w(p, q)=m^{2}\left(\left|p-p_{s_{i}}\right|^{2}+\left(p-p_{s_{i}}, q-q_{s_{i}}\right)+\left|q-q_{s_{i}}\right|^{2}\right)+O\left(\left|p-p_{s_{i}}\right|^{4}\right)+O\left(\left|q-q_{s_{i}}\right|^{4}\right)
$$

при $\left|p-p_{s_{i}}\right|,\left|q-q_{s_{i}}\right| \rightarrow 0, i=\overline{1, n}$, вытекает следующая

Лемма 5. Существуют числа $C_{1}, C_{2}, C_{3}>0 u \delta>0$ такие, что выполняются следующие неравенства:

$$
\begin{gathered}
C_{1}\left(\left|p-p_{s_{i}}\right|^{2}+\left|q-q_{s_{i}}\right|^{2}\right) \leqslant w(p, q) \leqslant C_{2}\left(\left|p-p_{s_{i}}\right|^{2}+\left|q-q_{s_{i}}\right|^{2}\right), \\
(p, q) \in U_{\delta}\left(p_{s_{i}}\right) \times U_{\delta}\left(q_{s_{i}}\right), \quad i=\overline{1, n}
\end{gathered}
$$

$u w(p, q) \geqslant C_{3}$ для любъх $p, q$, удовлетворяющих по крайней мере одному из условий $p \notin \bigcup_{i=1}^{n} U_{\delta}\left(p_{s_{i}}\right) u q \notin \bigcup_{i=1}^{n} U_{\delta}\left(q_{s_{i}}\right)$.

Лемма 6. Oператор $h_{\mu_{0}}(p), p \in \mathbf{T}^{3}$, не имеет отрицательных собственных значений. 
ДоказАтельство. Сначала покажем, что для любого $p \in \mathbf{T}^{3} \backslash\left\{p_{s_{1}}, p_{s_{2}}, \ldots, p_{s_{n}}\right\}$ имеет место неравенство $\Delta_{\mu_{0}}(p, 0)>\Delta_{\mu_{0}}\left(p_{s_{1}}, 0\right)$. Пусть

$$
\Lambda(p)=\int_{\mathbf{T}^{3}} \frac{\varphi^{2}(q) d q}{w(p, q)} .
$$

Тогда, используя свойства функции $w(\cdot, \cdot)$, легко можно проверить, что имеет место равенство $\Lambda\left(p_{s_{1}}\right)=\Lambda\left(p_{s_{i}}\right), i=\overline{2, n}$. Так как функции $\varphi(\cdot)$ и $w(\cdot, \cdot)$ четные, функция $\Lambda(\cdot)$ также является четной и, следовательно,

$$
\begin{aligned}
\Lambda(p)-\Lambda\left(p_{s_{1}}\right)= & \frac{1}{4} \int_{\mathbf{T}^{3}} \frac{2 w\left(p_{s_{1}}, q\right)-(w(p, q)+w(-p, q))}{w(p, q) w(-p, q) w\left(p_{s_{1}}, q\right)} \times \\
& \times(w(p, q)+w(-p, q)) \varphi^{2}(q) d q- \\
& -\frac{1}{4} \int_{\mathbf{T}^{3}} \frac{(w(p, q)+w(-p, q))^{2}}{w(p, q) w(-p, q) w\left(p_{s_{1}}, q\right)} \varphi^{2}(q) d q .
\end{aligned}
$$

В силу равенства

$$
w\left(p_{s_{1}}, q\right)-\frac{w(p, q)+w(-p, q)}{2}=\sum_{j=1}^{3}\left(\cos \left(m p^{(j)}\right)-1\right)\left(1+\cos \left(m q^{(j)}\right)\right)
$$

и формулы (9) имеем, что для любого $p \in \mathbf{T}^{3} \backslash\left\{p_{s_{1}}, p_{s_{2}}, \ldots, p_{s_{n}}\right\}$ справедливо неравенство $\Lambda(p)-\Lambda\left(p_{s_{1}}\right)<0$. Из определения числа $\mu_{0}(6)$ следует, что $\Delta_{\mu_{0}}\left(p_{s_{1}}, 0\right)=0$. Следовательно, $\Delta_{\mu_{0}}(p, z)>\Delta_{\mu_{0}}\left(p_{s_{1}}, 0\right)=0$ для любых $p \in \mathbf{T}^{3}$ и $z<0$. По лемме 1 это означает, что оператор $h_{\mu_{0}}(p), p \in \mathbf{T}^{3}$, не имеет отрицательных собственных значений. Лемма 6 доказана.

ЛЕмма 7. Функиия $f$, определенная соотношением (5), удовлетворяет уравнению $h_{\mu_{0}}\left(p_{s_{1}}\right) f=0$.

Доказательство. Сначала покажем, что $f \in L_{1}\left(\mathbf{T}^{3}\right) \backslash L_{2}\left(\mathbf{T}^{3}\right)$, т.е.

$$
\int_{\mathbf{T}^{3}}|f(q)| d q<\infty, \quad \int_{\mathbf{T}^{3}}|f(q)|^{2} d q=\infty .
$$

Вспомним, что $\Delta_{\mu_{0}}\left(p_{s_{1}}, 0\right)=0$. Множество $\left\{\left(p_{s_{i}}, q_{s_{i}}\right) \in\left(\mathbf{T}^{3}\right)^{2}: i=\overline{1, n}\right\}$ по построению таково, что $\varphi\left(q_{s_{i}}\right) \neq 0$ для всех $i=\overline{1, \mathbf{n}}$ и $\varphi\left(q_{s_{i}}\right)=0$ для $i=\overline{\mathbf{n}+1, n}$ (см. замечание 1). Учитывая эти факты и определение $(2)$ функции $\varepsilon(\cdot)$, получим, что существуют числа $C_{1}, C_{2}, C_{3}>0$ и $\delta>0$ такие, что справедливы неравенства

$$
\begin{array}{rlrl}
C_{1}\left|q-q_{s_{i}}\right|^{2} & \leqslant \varepsilon(q) \leqslant C_{2}\left|q-q_{s_{i}}\right|^{2}, & & q \in U_{\delta}\left(q_{s_{i}}\right), \quad i=\overline{1, n} \\
\varepsilon(q) & \geqslant C_{3}, & & q \in \mathbf{T}^{3} \backslash \bigcup_{i=1}^{n} U_{\delta}\left(q_{s_{i}}\right) ; \\
|\varphi(q)| \geqslant C_{3}, & & q \in U_{\delta}\left(q_{s_{i}}\right), \quad i=\overline{1, \mathbf{n}}
\end{array}
$$

а также (в случае $\mathbf{n}<n$ )

$$
C_{1}\left|q-q_{s_{i}}\right|^{2} \leqslant|\varphi(q)| \leqslant C_{2}\left|q-q_{s_{i}}\right|^{2}, \quad q \in U_{\delta}\left(q_{s_{i}}\right), \quad i=\overline{\mathbf{n}+1, n} .
$$


Используя последнее неравенство, имеем

$$
\begin{gathered}
\int_{\mathbf{T}^{3}}|f(q)| d q \leqslant C_{1} \sum_{j=1}^{n_{0}} \int_{U_{\delta}\left(q_{n_{j}}\right)} \frac{d q}{\left|q-q_{s_{j}}\right|^{2}}+C_{2}<\infty, \\
\int_{\mathbf{T}^{3}}|f(q)|^{2} d q \geqslant C_{1} \sum_{j=1}^{n_{0}} \int_{U_{\delta}\left(q_{n_{j}}\right)} \frac{d q}{\left|q-q_{s_{j}}\right|^{4}}+C_{2}=\infty .
\end{gathered}
$$

Легко можно проверить, что функция $f$ удовлетворяет уравнению $h_{\mu_{0}}\left(p_{s_{1}}\right) f=0$. Лемма 7 доказана.

\section{4. ПРИНЦИП БИРМАНА-ШВИНГЕРА}

Для любого ограниченного самосопряженного оператора $A$, действующего в гильбертовом пространстве $\mathcal{R}$, определим число

$$
d(\lambda, A)=\sup \{\operatorname{dim} F:(A u, u)>\lambda, u \in F \subset \mathcal{R},\|u\|=1\} .
$$

Число $d(\lambda, A)$ равно бесконечности, если $\lambda$ - внутренняя точка существенного спектра; если $d(\lambda, A)$ конечно, то оно равно числу собственных значений оператора $A$, больших $\lambda$. По определению числа $N_{\mu}(z)$ имеем, что $N_{\mu}(z)=d\left(-z,-H_{\mu}\right)$ при $-z>-\tau_{\mathrm{ess}}\left(H_{\mu}\right)$. Отметим, что $\Delta_{\mu}(p, z) \geqslant 0$ для любых $p \in \mathbf{T}^{3}$ и $z<\tau_{\mathrm{ess}}\left(H_{\mu}\right)$, следовательно, существует $\sqrt{\Delta_{\mu}(p, z)} \geqslant 0$.

В исследованиях дискретного спектра оператора $H_{\mu}$ основную роль играет компактный интегральный (симметризованный) оператор $T_{\mu}(z), z<\tau_{\mathrm{ess}}\left(H_{\mu}\right)$, действующий в $L_{2}\left(\mathbf{T}^{3}\right)$, с ядром

$$
\frac{\mu \varphi(p) \varphi(q)}{\sqrt{\Delta_{\mu}(p, z)} \sqrt{\Delta_{\mu}(q, z)}(w(p, q)-z)} .
$$

Следующая лемма отражает известный принцип Бирмана-Швингера для оператора $H_{\mu}$ (см. работы [3]-[5], [11]-[13]).

ЛЕмма 8. При всех $z<\tau_{\mathrm{ess}}\left(H_{\mu}\right)$ оператор $T_{\mu}(z)$ является компактным и непрерывным по $z$, и справедливо равенство $N_{\mu}(z)=d\left(1, T_{\mu}(z)\right)$.

Эта лемма доказана в работе [13] для несимметричного случая.

\section{5. ДОКАЗАТЕЛЬСТВО ОСНОВНОГО РЕЗУЛЬТАТА}

В этом разделе мы выводим асимптотику (7) для числа $N_{\mu_{0}}(z)$ собственных значений оператора $H_{\mu_{0}}$, лежащих левее $z<0$, и тем самым доказываем теорему 2. Доказательство теоремы основано на нескольких леммах и некоторых утверждениях из работ [3], [11].

Сначала найдем асимптотику $d\left(1, T_{\mu_{0}}(z)\right)$ при $z \rightarrow-0$. Для этого сведем анализ спектральных свойств оператора $T_{\mu_{0}}(z)$ к анализу спектральных свойств оператора $\mathbf{T}(r)$, который исследован в работе [11].

Верна следующая 
Лемма 9. Пусть $A(z)=A_{0}(z)+A_{1}(z)$, где оператор $A_{0}(z)$ (onepamop $A_{1}(z)$ ) является компактным и непрерывным при $z<0$ (соответственно, при $z \leqslant 0$ ) в сильной операторной топологии. Предположим, что для функции $f(\cdot), f(z) \rightarrow 0$ при $z \rightarrow 0$, имеет место равенство

$$
\lim _{z \rightarrow-0} f(z) d\left(\gamma, A_{0}(z)\right)=l(\gamma)
$$

где функция $l(\cdot)$ определена и непрерывна на полуоси $(0 ;+\infty)$. Тогда аналогичныи предел существует для $A(z)$ :

$$
\lim _{z \rightarrow-0} f(z) d(\gamma, A(z))=l(\gamma)
$$

Для доказательства леммы 9 см. лемму 4.9 из работы [3].

Пусть $T(\delta ;|z|)$ - интегральный оператор, действующий в $L_{2}\left(\mathbf{T}^{3}\right)$, с ядром

$$
\frac{1}{2 \pi^{2}} \sum_{i=1}^{\mathbf{n}} \frac{\chi_{\delta}\left(p-p_{s_{i}}\right) \chi_{\delta}\left(q-q_{s_{i}}\right)\left(\frac{3}{4}\left|p-p_{s_{i}}\right|^{2}+|z|\right)^{-1 / 4}\left(\frac{3}{4}\left|q-q_{s_{i}}\right|^{2}+|z|\right)^{-1 / 4}}{\left|p-p_{s_{i}}\right|^{2}+\left(p-p_{s_{i}}, q-q_{s_{i}}\right)+\left|q-q_{s_{i}}\right|^{2}+|z|} .
$$

Здесь $\chi_{\delta}(\cdot)$ - характеристическая функция множества $U_{\delta}(0)$. Оператор $T(\delta ;|z|)$ называется сингулярной частью оператора $T_{\mu_{0}}(z)$.

ЛЕмма 10. Для любых $z \leqslant 0$ при малых $\delta>0$ разность $T_{\mu_{0}}(z)-T(\delta ;|z|)$ является оператором Гилъберта-Шмидта, непрерывным в сильной операторной топологии в точке $z=0$.

ДокАЗАТЕЛЬство. На основании лемм $3-5$ имеем, что существуют числа $C_{1}, C_{2}>0$ такие, что ядро оператора $T_{\mu_{0}}(z)-T(\delta ;|z|)$ оценивается функцией

$$
C_{1}+C_{2} \sum_{i=1}^{\mathbf{n}} \frac{\left|p-p_{s_{i}}\right|^{-1 / 2}+\left|q-q_{s_{i}}\right|^{-1 / 2}+1}{\left|p-p_{s_{i}}\right|^{2}+\left(p-p_{s_{i}}, q-q_{s_{i}}\right)+\left|q-q_{s_{i}}\right|^{2}},
$$

следовательно, оператор $T_{\mu_{0}}(z)-T(\delta ;|z|)$ является оператором Гильберта-Шмидта при всех $z \leqslant 0$, непрерывным в сильной операторной топологии в точке $z=0$. Лемма 10 доказана.

Пространство функций $f$, носители которых лежат в $\bigcup_{i=1}^{\mathbf{n}} U_{\delta}\left(p_{s_{i}}\right)$, является инвариантным подпространством оператора $T(\delta ;|z|)$. Пусть $T_{0}(\delta ;|z|)$ - сужение опеpaтора $T(\delta ;|z|)$ на это подпространство, т.е. интегральный оператор, действующий в гильбертовом пространстве $L_{2}\left(\bigcup_{i=1}^{\mathbf{n}} U_{\delta}\left(p_{s_{i}}\right)\right)$, с ядром

$$
\frac{1}{2 \pi^{2}} \sum_{i=1}^{\mathbf{n}} \frac{\chi_{\delta}\left(q-q_{s_{i}}\right)\left(\frac{3}{4}\left|p-p_{s_{i}}\right|^{2}+|z|\right)^{-1 / 4}\left(\frac{3}{4}\left|q-q_{s_{i}}\right|^{2}+|z|\right)^{-1 / 4}}{\left|p-p_{s_{i}}\right|^{2}+\left(p-p_{s_{i}}, q-q_{s_{i}}\right)+\left|q-q_{s_{i}}\right|^{2}+|z|} .
$$

Обозначим через $\operatorname{diag}\left\{A_{1}, A_{2}, \ldots, A_{\mathbf{n}}\right\}$ диагональный матричный оператор размера $\mathbf{n} \times \mathbf{n}$ с диагональными элементами $A_{1}, A_{2}, \ldots, A_{\mathbf{n}}$.

В силу равенства $L_{2}\left(\bigcup_{i=1}^{\mathbf{n}} U_{\delta}\left(p_{s_{i}}\right)\right)=\bigoplus_{i=1}^{\mathbf{n}} L_{2}\left(U_{\delta}\left(p_{s_{i}}\right)\right)$ оператор $T_{0}(\delta ;|z|)$ записывается как диагональная матрица:

$$
T_{0}(\delta ;|z|)=\operatorname{diag}\left\{T_{0}^{(1)}(\delta ;|z|), T_{0}^{(2)}(\delta ;|z|), \ldots, T_{0}^{(\mathbf{n})}(\delta ;|z|)\right\},
$$


где $T_{0}^{(i)}(\delta ;|z|)$ есть интегральный оператор, действующий в $L_{2}\left(U_{\delta}\left(p_{s_{i}}\right)\right)$, ядро которого совпадает с $i$-м слагаемым в $(10), i=\overline{1, \mathbf{n}}$.

Пусть

$$
L_{2}^{(\mathbf{n})}\left(U_{r}(0)\right)=\left\{f=\left(f_{1}, f_{2}, \ldots, f_{\mathbf{n}}\right): f_{i} \in L_{2}\left(U_{r}(0)\right), i=\overline{1, \mathbf{n}}\right\} .
$$

Легко можно показать, что оператор $T_{0}(\delta ;|z|)$ унитарно эквивалентен оператору $T_{1}(r), r=|z|^{-1 / 2}$, действующему в $L_{2}^{(\mathbf{n})}\left(U_{r}(0)\right)$ по формуле

$$
T_{1}(r)=\operatorname{diag}\left\{T_{1}^{(1)}(r), T_{1}^{(2)}(r), \ldots, T_{1}^{(\mathbf{n})}(r)\right\}
$$

где $T_{1}^{(i)}(r)$ есть интегральный оператор в $L_{2}\left(U_{r}(0)\right)$ с ядром

$$
\frac{1}{2 \pi^{2}} \frac{\chi_{r}(q)}{\left(\frac{3}{4}|p|^{2}+1\right)^{1 / 4}\left(\frac{3}{4}|q|^{2}+1\right)^{1 / 4}\left(|p|^{2}+(p, q)+|q|^{2}+1\right)} .
$$

Отметим, что унитарная эквивалентность этих операторов осуществляется при помощи унитарного оператора

$$
B_{r}=\operatorname{diag}\left\{B_{r}^{(1)}, B_{r}^{(2)}, \ldots, B_{r}^{(\mathbf{n})}\right\}: \bigoplus_{i=1}^{\mathbf{n}} L_{2}\left(U_{\delta}\left(p_{s_{i}}\right)\right) \rightarrow L_{2}^{(\mathbf{n})}\left(U_{r}(0)\right),
$$

где действие оператора $B_{r}^{(i)}: L_{2}\left(U_{\delta}\left(p_{s_{i}}\right)\right) \rightarrow L_{2}\left(U_{r}(0)\right)$ задается формулой

$$
\left(B_{r}^{(i)} f\right)(p)=\left(\frac{r}{\delta}\right)^{-3 / 2} f\left(\frac{\delta}{r}\left(p-p_{s_{i}}\right)\right), \quad i=\overline{1, \mathbf{n}}
$$

Так как пространство $L_{2}^{(\mathbf{n})}\left(U_{r}(0)\right)$ изоморфно $L_{2}\left(U_{r}(0)\right)$, мы перепишем оператор $T_{1}(r)$ как интегральный оператор в $L_{2}\left(U_{r}(0)\right)$ с ядром

$$
\frac{\mathbf{n}}{2 \pi^{2}} \frac{\chi_{r}(q)}{\left(\frac{3}{4}|p|^{2}+1\right)^{1 / 4}\left(\frac{3}{4}|q|^{2}+1\right)^{1 / 4}\left(|p|^{2}+(p, q)+|q|^{2}+1\right)} .
$$

Далее, заменяя

$$
\left(\frac{3}{4}|p|^{2}+1\right)^{1 / 4}, \quad\left(\frac{3}{4}|q|^{2}+1\right)^{1 / 4}
$$

на

$$
\left(\frac{3}{4}|p|^{2}\right)^{1 / 4}\left(1-\chi_{1}(p)\right)^{-1}, \quad\left(\frac{3}{4}|q|^{2}\right)^{1 / 4}\left(1-\chi_{1}(q)\right)^{-1}
$$

соответственно, мы получим оператор $T_{2}(r)$. Разность $T_{1}(r)-T_{2}(r)$ является оператором Гильберта-Шмидта, непрерывным в точке $z=0$ в сильной операторной топологии.

Пространство функций $f$, носители которых лежат в $L_{2}\left(U_{r}(0) \backslash U_{1}(0)\right)$, является инвариантным подпространством оператора $T_{2}(r)$. Ядро этого оператора имеет следующий вид:

$$
K_{\mathbf{n}}(p, q)=\frac{\mathbf{n}}{\sqrt{3} \pi^{2}} \frac{1-\chi_{1}(q)}{|p|^{1 / 2}|q|^{1 / 2}\left(|p|^{2}+(p, q)+|q|^{2}\right)} .
$$


Пусть $\mathbf{T}(r)$ - интегральный оператор, действующий в $L_{2}\left(U_{r}(0) \backslash U_{1}(0)\right)$, с ядром $K_{2}(p, q)$. Следующая лемма доказана в работе [11].

Лемма 11. Имеет место равенство

$$
\lim _{z \rightarrow-0} \frac{d(1, \mathbf{T}(z))}{|\ln | z||}=\frac{\gamma_{0}}{2 \pi},
$$

где $\gamma_{0}$ является единственным положительным корнем уравнения (8).

Доказательство теоремы 2 следует из лемм 8-11.

Благодарности. Автор выражает благодарность рецензенту за ценные и полезные замечания. Автор также благодарит Международный центр теоретической физики им. Абдуса Салама (Италия, Триест) за гостеприимство и поддержку и Международное математическое общество за дорожный грант.

\section{Список литературы}

[1] В. Ефимов, ЯФ, 12 (1970), 1080-1091.

[2] Д. Р. Яфаев, Матем. сб., 94(136):4(8) (1974), 567-593.

[3] A. V. Sobolev, Comm. Math. Phys., 156:1 (1993), 101-126.

[4] H. Tamura, Nagoya Math. J., 130 (1993), 55-83.

[5] X. P. Wang, J. Func. Anal., 209:1 (2004), 137-161.

[6] D. C. Mattis, Rev. Modern Phys., 58:2 (1986), 361-379.

[7] A. I. Mogilner, "The problem of a few quasi-particles in solid-state physics", Applications of Self-Adjoint Extensions in Quantum Physics, Lecture Notes in Phys., 324, eds. P. Exner, P. Šeba, 1989, 160-173.

[8] С. Н. Лакаев, ТМФ, 89:1 (1991), 94-104.

[9] С. Н. Лакаев, Функи. анализ и его прил., 27:3 (1993), 15-28.

[10] С. Н. Лакаев, М.Э. Муминов, ТМФ, 135:3 (2003), 478-503.

[11] Ж. И. Абдуллаев, С. Н. Лакаев, ТМФ, 136:2 (2003), 231-245.

[12] S. Albeverio, S. N. Lakaev, Z. I. Muminov, Ann. Henri Poincaré, 5:4 (2004), 743-772.

[13] S. Albeverio, S. N. Lakaev, Z. I. Muminov, Russ. J. Math. Phys., 14:4 (2007), 377-387.

[14] М. Рид, Б. Саймон, Методы современной математической физики. Т. 4. Анализ операторов, Мир, М., 1982.

Поступила в редакцию 2.06.2009, после доработки 9.10.2009 